\title{
A HIGH-MASS PROTOBINARY SYSTEM WITH SPATIALLY RESOLVED CIRCUMSTELLAR ACCRETION DISKS AND CIRCUMBINARY DISK
}

\author{
S. Kraus ${ }^{1}$, J. Kluska ${ }^{1}$, A. Kreplin ${ }^{1}$, M. Bate ${ }^{1}$, T.J. Harries ${ }^{1}$, K.H. Hofmann ${ }^{2}$, E. Hone ${ }^{1}$, J.D. Monnier ${ }^{3}$, \\ G. WeigelT ${ }^{2}$, W.J. DE Wit ${ }^{4}$, M. WitTkOWski ${ }^{5}$ \\ 1 School of Physics, Astrophysics Group, University of Exeter, Stocker Road, Exeter EX4 4QL, UK \\ 2 Max-Planck-Institut für Radioastronomie, Auf dem Hügel 69, 53121 Bonn, Germany \\ 3 Department of Astronomy, University of Michigan, 311 West Hall, 1085 South University Ave, Ann Arbor, MI 48109, USA \\ 4 ESO, Alonso de Cordova 3107, Vitacura, Santiago 19, Chile \\ 5 ESO, Karl-Schwarzschild-Str. 2, 85748 Garching bei München, Germany \\ Draft version December 8, 2016
}

\begin{abstract}
High-mass multiples might form via fragmentation of self-gravitational disks or alternative scenarios such as disk-assisted capture. However, only few observational constraints exist on the architecture and disk structure of high-mass protobinaries and their accretion properties. Here we report the discovery of a close $(57.9 \pm 0.2 \mathrm{mas}=170 \mathrm{au})$ high-mass protobinary, IRAS17216-3801, where our VLTI/GRAVITY + AMBER near-infrared interferometry allows us to image the circumstellar disks around the individual components with $\sim 3$ milliarcsecond resolution. We estimate the component masses to $\sim 20$ and $\sim 18 M_{\odot}$ and find that the radial intensity profiles can be reproduced with an irradiated disk model, where the inner regions are excavated of dust, likely tracing the dust sublimation region in these disks. The circumstellar disks are strongly misaligned with respect to the binary separation vector, which indicates that the tidal forces did not have time to realign the disks, pointing towards a young dynamical age of the system. We constrain the distribution of the $\operatorname{Br} \gamma$ and COemitting gas using VLTI/GRAVITY spectro-interferometry and VLT/CRIRES spectro-astrometry and find that the secondary is accreting at a higher rate than the primary. VLT/NACO imaging shows $L^{\prime}$-band emission on $3-4 \times$ larger scales than the binary separation, matching the expected dynamical truncation radius for the circumbinary disk. The IRAS17216-3801 system is $\sim 3 \times$ more massive and $\sim 5 \times$ more compact than other high-mass multiplies imaged at infrared wavelengths and the first high-mass protobinary system where circumstellar and circumbinary dust disks could be spatially resolved. This opens exciting new opportunities for studying star-disk interactions and the role of multiplicity in high-mass star formation.
\end{abstract}

Subject headings: stars: formation — binaries: close — stars: massive — stars: individual (IRAS172163801) — accretion, accretion disks — techniques: interferometric

\section{INTRODUCTION}

There is now solid evidence that the formation of highmass stars $\left(>10 M_{\odot}\right)$ involves accretion through circumstellar disks (Cesaroni et al. 2005; Kraus et al. 2010; Boley et al. 2013; Johnston et al. 2015). This observational evidence supports recent theoretical work which suggests that the radiation pressure barrier problem can be overcome when considering more complex than spherically symmetric infall geometries (Yorke \& Sonnhalter 2002; Krumholz et al. 2009). However, the classical accretion disk scenario, which assumes the monolithic collapse of a core to a single star and which is commonly applied to low-mass star formation, fails to explain the high multiplicity fraction that has been observed for high-mass stars. Multiplicity studies on main-sequence stars found that $\gtrsim 80 \%$ of all O-type stars $\left(M \gtrsim 16 M_{\odot}\right)$ are close multiple systems, while this fraction rapidly drops to $20 \%$ for stellar masses of $\sim 3 M_{\odot}$ (Chini et al. 2012). At the same time, the number of companions per system also increases with stellar mass. For instance, the five O-/early B-type stars in the Orion Trapezium cluster have on av-

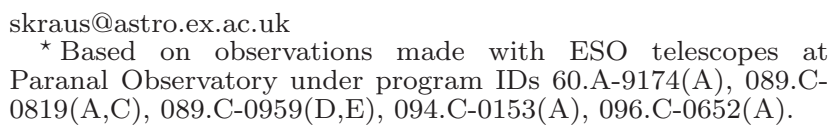

* Based on observations made with ESO telescopes at Paranal Observatory under program IDs 60.A-9174(A), 089.C0819(A,C), 089.C-0959(D,E), 094.C-0153(A), 096.C-0652(A).

erage 2.5 known companions, which is about $5 \times$ higher than what has been found for low-mass stars (Grellmann et al. 2013). Different mechanisms have been proposed in order to explain these extraordinary characteristics. For instance, these high-mass multiples might form via fragmentation of self-gravitating massive disks (Kratter \& Matzner 2006), disk-assisted capture (Clarke \& Pringle 1991; Bally \& Zinnecker 2005), or through failed mergers in stellar collisions (Dale \& Davies 2006). Testing these scenarios requires the detection of high-mass "protobinaries" that are still in their formation phase.

Here we report high-angular resolution observations that resolve the high-mass protostar IRAS17216-3801 into a close $(\sim 170 \mathrm{au})$ binary, where both components are associated with actively accreting circumstellar disks. IRAS17216-3801 (=G350.011-01.341) was identied as a high-mass YSO by Persson \& Campbell (1987) and its distance estimated to $3.08 \pm 0.6 \mathrm{kpc}$ (Boley et al. 2013). Various studies reported the detection of $\mathrm{OH}$ masers (Cohen et al. 1988; Argon et al. 2000; Caswell 1998). Boley et al. (2013) resolved the mid-infrared $(8-13 \mu \mathrm{m})$ emitting-region and found an elongated structure with a Gaussian full-width-at-half-maximum (FWHM) size of $\sim 85_{-33}^{+20}$ milliarcseconds (mas) and speculated that the emitting structure might be associated with a circum- 
stellar disk or an outflow.

We observed the source as part of a small survey on high-mass YSOs that we conducted with the Very Large Telescope Interferometer (VLTI) and its AMBER beam combiner. The AMBER measurements with low spectral resolution $(R=\lambda / \Delta \lambda=35)$ were complemented with higher spectral resolution data $(R=500)$ from the newly-commissioned VLTI/GRAVITY combiner.

\section{OBSERVATIONS}

\subsection{VLTI/AMBER spectro-interferometry}

We observed IRAS17216-3801 on 2012-05-06, 201206-01, and 2012-06-03 using the AMBER instrument (Petrov et al. 2007). The observations used the UT1UT2-UT4 and UT2-UT3-UT4 triplets of the VLTI array of four $8.2 \mathrm{~m}$ unit telescopes, with projected baseline lengths $30 \ldots 130 \mathrm{~m}$ (Fig. 1, top-left).

Our setup covered the $K$-band with $R=35$, where we employed a $50 \mathrm{~ms}$ integration time. The science star observations were bracketed with observations on calibrator stars with known diameters, which allowed us to correct for atmospheric and instrumental effects. As calibrators we selected HD155259 (2012-05-06, 2012-06$01, \mathrm{UDD}=0.226 \pm 0.016 \mathrm{mas})$ and HD165787 (2012-05-06, 2012-06-03, UDD $=0.49 \pm 0.04 \mathrm{mas})$. The uniform disk diameters (UDD) were estimated using the JMMC SearchCal tool (Bonneau et al. 2011).

The visibilities and closure phases were extracted using the amdlib software (Release 3.0.4; Tatulli et al. 2007; Chelli et al. 2009). The resulting amplitudes/phases show strong wavelength-differential modulations (Fig. 2, bottom), which is characteristic for wide-separation multiple systems. Furthermore, we noticed that the observables, in particular the closure phases, changed slightly between individual object exposures. Therefore, we decided to analyse the individual exposures $(\sim 50 \mathrm{~s})$ separately in order to avoid temporal smearing. We also checked whether our data might be affected by bandwidth smearing effects using the procedure outlined in Kraus et al. (2005). We estimate the loss of coherence to $\lesssim 2.5 \%$ for our longest baseline, which is within our measurement uncertainty.

\subsection{VLTI/GRAVITY spectro-interferometry}

On 2016-06-23 we acquired observations with the VLTI/GRAVITY instrument (Eisenhauer et al. 2011, Eisenhauer et al., in prep.) as part of Science Verification. The observations combined the light from the four VLTI $1.8 \mathrm{~m}$ auxiliary telescopes and covered the $K$-band with $R=500$. The telescopes were in the compact configuration (A0-B2-D0-C1) with projected baseline lengths $11 \ldots 34 \mathrm{~m}$. Seven object exposures were recorded on IRAS17216-3801, where each exposure consists of 30 interferograms taken with 10s integration time.

The science star observations were bracketed with observations on calibrator HD159868 (UDD $=0.358 \pm$ $0.025 \mathrm{mas})$ Wavelength-differential visibilities and phases were extracted using the GRAVITY pipeline (Release 0.8.4; Lapeyrere et al. 2014). Over the 66 minutes covered by our data recording sequence, we see strong visibility and phase changes. Therefore, we splitted the data into bins and reduced them separately, where we use 50 s binning to minimize potential temporal smear- ing effects in our model fits.

\subsection{VLT/NACO imaging}

We imaged the system on 2015-07-25 with the VLT/NACO adaptive optics system (Lenzen et al. 2003; Rousset et al. 2003) using a $J$-band filter $(8 \times 30$ s integration) and a selection of narrowband filters centered on the [Fe II] shock-tracing line (NB1.64; plus adjacent continuum with NB1.75 filter; $20 \times 1$ s integration) and on the $\mathrm{H}_{2}$ line (NB2.12; plus continuum with NB2.17 filter; $250 \times 1$ s integration). For the narrowband filters, we interlayed the individual pointings with calibrator observations on HD155974 to determine the point spread function. The system was also observed with a $L^{\prime}$-band filter during our 2016-03-28 run ( $1500 \times 0.2$ s integration). To eliminate the thermal background, we dithered the object between the different detector quadrants. The images were reduced using the NACO pipeline (Release 4.4.1). The continuum-subtracted [Fe II] and $\mathrm{H}_{2}$ images do not exhibit extended line emission and are therefore not further discussed.

\subsection{VLT/CRIRES spectro-astrometry}

We obtained VLT/CRIRES high-resolution spectroscopy (Kaeufl et al. 2004) near the $\operatorname{Br} \gamma 2.166 \mu \mathrm{m}$ line on 2012-07-05. The long-slit spectra offer spectral resolution $R=100,000$. Our setup was optimized for the spectro-astrometry technique, where we measure the centroid position along the slit direction with high precision in order to derive the photocenter offset in the line with respect to the continuum. We recorded spectra for six slit orientations $\left(\mathrm{PA}=0,60,120^{\circ}\right.$ plus anti-parallel orientations; each with $4 \times 45$ s integrations), which allows us to remove spurious instrumental effects. Spectra were recorded on the calibrator Hip88154 to correct for telluric lines. Details of our data processing procedure and on our spectro-astrometric observable (X) can be found in Kraus et al. (2012).

\section{APERTURE SYNTHESIS IMAGING}

To derive the brightness distribution from the VLTI data in a model-independent fashion we used the IRBis image reconstruction algorithm (Hofmann et al. 2014). This algorithm minimizes a global cost function that includes a likelihood term (which determines the likelihood that the image is a representation of the data) plus a regularisation term that prevents the algorithm from overfitting the data in the presence of an incomplete $u v$ coverage.

We first apply the algorithm to the GRAVITY data alone. The resulting image (Fig. 1, bottom-left) shows a binary with separation $\sim 58$ mas $(170 \mathrm{au}$ at $3.1 \mathrm{kpc}$ ) and a $K$-band contrast of $\sim 0.6$.

We were also able to reconstruct an image from the combined 2016/GRAVITY and 2012/AMBER data set, after accounting for the orbital motion between the two epochs. For this purpose, we use the method outlined in Kraus et al. (2005), where the $u v$-plane is rotated and scaled synchronously to the system motion. In order to quantify the separation change $\Delta \rho$ and position angle (PA) change $\Delta \theta$ between 2012 and 2016, we varied these parameters systematically on a grid and searched for the image reconstructions with the lowest $\chi^{2}$. We find a 
TABLE 1

Model-fitting Results (SECT. 4)

\begin{tabular}{|c|c|c|c|c|}
\hline & & & GAUSS model & DISK model \\
\hline Binary separation & $\rho$ & [mas] & $57.94 \pm 0.24$ & $57.93 \pm 0.16$ \\
\hline Binary PA & $\Theta$ & {$\left[{ }^{\circ}\right]$} & $166.8 \pm 0.2$ & $166.76 \pm 0.2$ \\
\hline PA change 2012-2016 & $\Delta \Theta$ & {$[0]$} & $7 \pm 2$ & $7 \pm 2$ \\
\hline Flux ratio & $F_{\mathrm{A}} / F_{\mathrm{B}}$ & & $1.29 \pm_{-0.02}^{+0.09}$ & $1.26 \pm_{-0.02}^{+0.13}$ \\
\hline Extended flux contribution & $F_{\text {ext }} / F_{\text {tot }}$ & & $0.16 \pm 0.09$ & $0.16 \pm 0.06$ \\
\hline \multicolumn{5}{|c|}{ Circumprimary disk, continuum (Northern component, $\mathbf{A}$ ) } \\
\hline Contribution extended flux & $F_{\mathrm{A}}^{\mathrm{cs}} / F_{\mathrm{A}}$ & & $0.60 \pm 0.08$ & $0.64 \pm 0.04$ \\
\hline Disk inclination & $i_{\mathrm{A}}$ & {$\left[^{\circ}\right]$} & $89 \pm 10$ & $60 \pm 10$ \\
\hline Disk PA & $\theta_{\mathrm{A}}$ & [0] & $64 \pm 9$ & $67 \pm 7$ \\
\hline Gaussian FWHM size & $\Sigma_{\mathrm{A}}$ & [mas] & $7.63 \pm 0.8$ & - \\
\hline Inner disk radius & $r_{\mathrm{A}}^{\mathrm{in}}$ & [mas] & - & $2.77 \pm 0.39$ \\
\hline Outer disk radius & $r_{\mathrm{A}}^{\text {out }}$ & [mas] & - & $>12$ \\
\hline \multicolumn{5}{|c|}{ Circumsecondary disk, continuum (Southern component, B) } \\
\hline Contribution extended flux & $F_{\mathrm{B}}^{\mathrm{cs}} / F_{\mathrm{B}}$ & & $0.90 \pm 0.05$ & $0.96 \pm 0.09$ \\
\hline Disk inclination & $i_{\mathrm{B}}$ & {$\left[{ }^{\circ}\right]$} & $42 \pm 7$ & $38 \pm 10$ \\
\hline Disk PA & $\theta_{\mathrm{B}}$ & {$[0]$} & $185 \pm 32$ & $159 \pm 15$ \\
\hline Gaussian FWHM size & $\Sigma_{\mathrm{B}}$ & [mas] & $4.60 \pm 0.4$ & - \\
\hline Inner disk radius & $r_{\mathrm{B}}^{\mathrm{in}}$ & [mas] & - & $2.49 \pm 0.42$ \\
\hline \multirow[t]{2}{*}{ Outer disk radius } & $r_{\mathrm{B}}^{\mathrm{Out}}$ & [mas] & - & $>10$ \\
\hline & $\chi_{\mathrm{r}}^{2}$ & & 1.71 & 1.11 \\
\hline
\end{tabular}

NOTE.
North.

$\chi^{2}$-minimum with a anti-clockwise rotation of $\Delta \theta=8.5^{\circ}$ between the two epochs, while the separation stayed constant within the uncertainties. This procedure results in the image shown in Fig. 1 (bottom-right) that represents the IRAS17216-3801 system at the 2016 epoch with a $3 \times 5$ mas beam

The binary is also detected in the NACO adaptive optics images obtained with the $J$-band, NB1.64, NB1.75, NB2.12, and NB2.17 filters, where we see some indications that the contrast increases towards shorter wavelengths, indicating that the northern component has a redder color than the southern component (Fig. 3).

In all images, the northern component appears brighter than the southern component, which leads us to denote the northern component with IRAS17216-3801- "A" and the southern component with "B". Comparing the intensity profile of the binary components in the image with the interferometric beam clearly indicates that the emission is spatially extended, both in the GRAVITY and GRAVITY+AMBER image (Fig. 1). The strong elongation seen in both components indicates that the circumstellar disks are seen under inclination. The extended flux around $\mathrm{A}$ is elongated along $\mathrm{PA} \sim 70^{\circ}$, while $\mathrm{B}$ is elongated along $\mathrm{PA} \sim 0^{\circ}$. Some artefacts are visible in the AMBER+GRAVITY images that result from the remaining imperfections in the $u v$-coverage. These artefacts reach up to $10 \%$ level of peak intensity, but are easy to discern, as they mainly cause a repetition of the source binary pattern along the south-east/north-west direction.

The emission from the circumstellar disks might also have been traced by VLTI/MIDI interferometry (Boley et al. 2013). This data could be fitted with an overresolved flux component ( $52 \%$ of total flux) plus an elongated component with a Gaussian FWHM of $85_{-33}^{+20}$ mas oriented along $\mathrm{PA}=125_{-10}^{+40} \circ$ (inclination $59_{-11}^{+5}{ }^{\circ}$, Boley et al. 2013). Within the large uncertainties, the size and orientation are consistent with the derived binary separation vector, which leads us to suggest that the compact MIDI component traced IRAS17216-3801-A+B, but did not fully resolve the components.

\section{MODELING}

\subsection{Spectral energy distribution}

To assist our further interpretation, we estimated the fundamental parameters of IRAS17216-3801 by compiling its spectral energy distribution (SED) using archival data. Integrating over the SED yields a bolometric luminosity $L_{\text {bol }}=6.1 \times 10^{4} \mathrm{~L}_{\odot}($ assuming $\mathrm{d}=3.08 \mathrm{kpc}$; Boley et al. 2013).

We then fitted the SED with the radiative transfer model grid by Robitaille et al. (2006) and find a bestfit model (ID \#3009730) with an age of $\sim 10^{5} \mathrm{yrs}$, a $24 M_{\odot}$ stellar mass and a massive disk (gas+dust mass $\left.1.2 \times 10^{-2} M_{\odot}\right)$ seen at intermediate inclination $\left(50^{\circ}\right)$. This SED model does not incorporate the companion, but we assume that it still provides a reasonable firstorder estimate, as the primary will dominate the bolometric luminosity. In order to estimate the mass of the individual components, we assume that the measured $K$ band contrast is representative for the bolometric luminosity split between the two components and estimate from the mass-luminosity relation $\left(L \propto M^{3}\right)$ the primary mass to $20 M_{\odot}$ and the secondary mass to $18 M_{\odot}$. Spatially resolved multi-wavelength observations will be necessary to improve on this very rough estimate.

\subsection{Structure of circumstellar+circumbinary disks (continuum)}

After identifying the basic morphology of the source with aperture synthesis imaging, we fit geometric models to the visibilities/phases, with the goal to determine the precise binary astrometry and to characterize the circumstellar emission. 

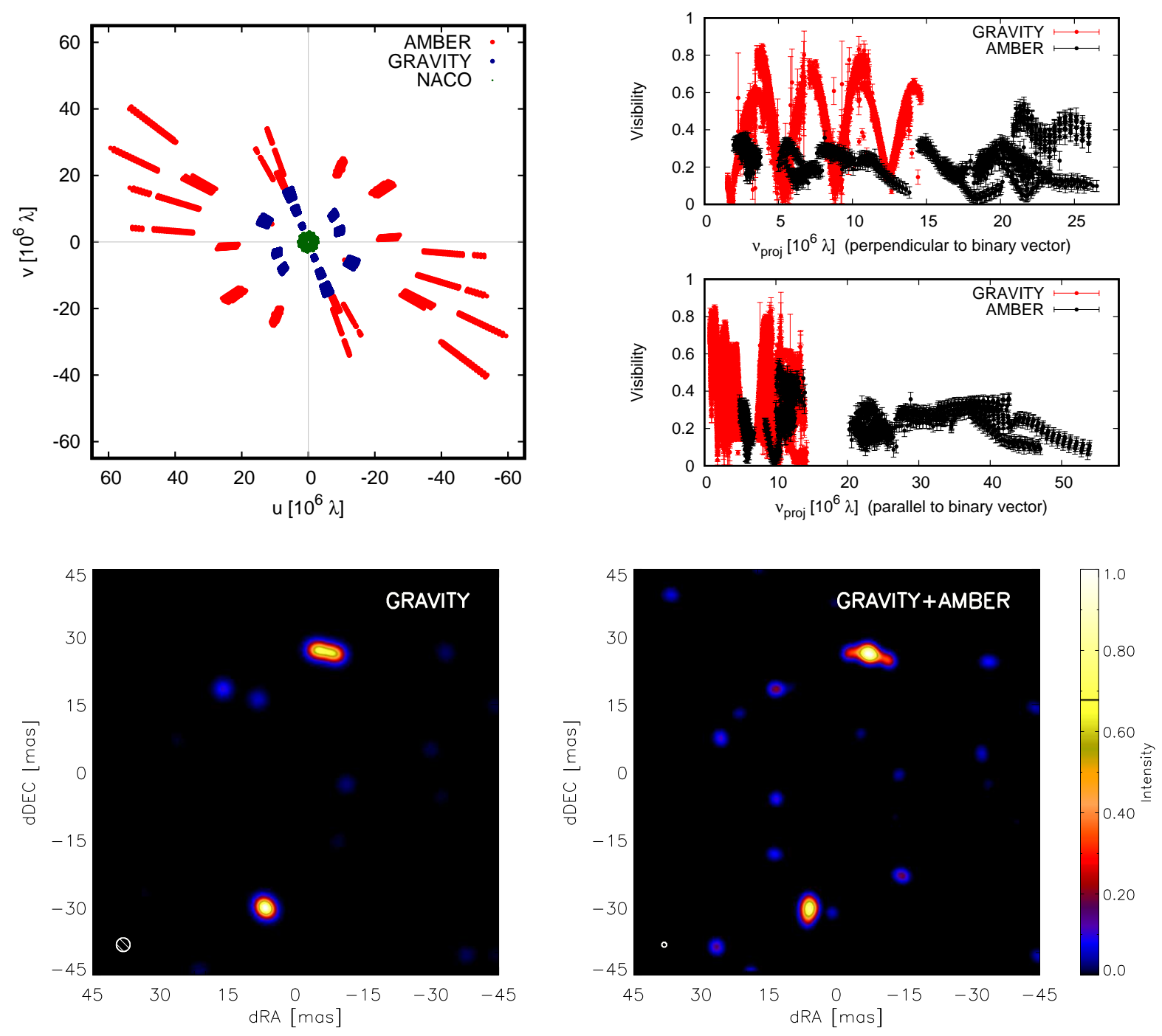

FIG. 1. - Spectro-interferometry on IRAS17216-3801: Obtained $u v$-coverage (top-left) and the measured visibility amplitudes, plotted as function of spatial frequency projected perpendicular (top-right, upper panel) and parallel to the binary separation vector (top-right, lower panel). Bottom: Aperture synthesis images obtained with GRAVITY (bottom-left) and GRAVITY+AMBER (bottom-right). 
Our model includes emission from two point sources (separation $\rho$; PA $\Theta$ ), where we allow the algorithm to associate each component with spatially extended flux. We denote the contributions of the spatially extended circumstellar emission to the integrated flux of the northern and southern component with $F_{\mathrm{A}}^{\mathrm{cs}} / F_{\mathrm{A}}$ and $F_{\mathrm{B}}^{\mathrm{cs}} / F_{\mathrm{B}}$, respectively. The flux ratio between the integrated fluxes is given by $F_{\mathrm{A}} / F_{\mathrm{B}}$. In addition, we allow the algorithm to attribute a fraction of the total flux $\left(F_{\text {ext }} / F_{\text {tot }}\right)$ to an over-resolved emission component.

We then fit the GRAVITY and AMBER data simultaneously (using 50s temporal binning), where we allow for orbital motion $(\Delta \Theta)$ between the two epochs (2012.378 and 2016.476). We explore the parameter space using an Levenberg-Marquardt algorithm and vary the initial guess parameters on a grid to avoid local minima.

For the brightness distribution of the circumstellar components, we assumed either elongated Gaussians ("GAUSS" model) or a temperature-power law disk model ("DISK" model). The GAUSS model introduces three free parameters to define the geometry of the circumstellar emission for each component, namely the inclination $\left(i_{\mathrm{A} ; \mathrm{B}}\right)$, the PA $\left(\theta_{\mathrm{A} ; \mathrm{B}}\right)$, and the full-width-athalf-maximum $\left(\Sigma_{\mathrm{A} ; \mathrm{B}}\right)$. The DISK model introduces four free parameters for each component, namely disk inclination, disk PA, and the inner and outer disk truncation radius $\left(r_{\mathrm{A} ; \mathrm{B}}^{\mathrm{in}}, r_{\mathrm{A} ; \mathrm{B}}^{\text {out }}\right)$. The radial temperature gradient of the disk is parameterized as $T(r)=1500 \mathrm{~K} \cdot T\left(r_{\text {in }}\right)^{-0.43}$, where $1500 \mathrm{~K}$ corresponds to the commonly assumed Silicate dust sublimation temperature and $r^{-0.43}$ to the theoretically derived temperature gradient for irradiated flared dust disks (Chiang \& Goldreich 1997). Our DISK model implementation has already been successfully applied to fit interferometry and SED data of intermediatemass and high-mass YSOs (Kraus et al. 2009, 2010).

The best-fit model (illustrated in Fig. 2, top-right) indicates that the disk around the southern component $\left(\theta_{\mathrm{B}}=159 \pm 15^{\circ}\right)$ is roughly aligned with the binary separation vector $\left(\Theta=166.76^{\circ}\right)$, while the northern component is oriented roughly perpendicular to it $\left(\theta_{\mathrm{A}}=67 \pm 7^{\circ}\right)$. For the radial intensity profile, we find that the DISK model provides a better representation of the data $\left(\chi_{\mathrm{r}}^{2}=1.11\right)$ than the GAUSS model $\left(\chi_{\mathrm{r}}^{2}=1.71\right)$, indicating that the disks are not smooth, but exhibit a central brightness depression, potentially tracing the opacity drop associated with dust sublimation near the star. The measured inner disk truncation radii of 2.78 and $2.49 \mathrm{mas}$ ( 8.6 and $7.7 \mathrm{au}$ ) are consistent with the theoretical dust sublimation radii, if one assumes grey dust and dust sublimation temperature of $1300 \mathrm{~K}$.

Comparing the properties of component A and B, we find that component A contributes a larger fraction to the total $K$-band flux, has a larger inner disk cavity, and a higher point-source flux contribution, all pointing to the conclusion that this is the more luminous and more massive component in the system.

Our best-fit model attributes $66 \%$ of the total flux to the circumstellar disks, $17 \pm 4 \%$ and $1 \pm 6 \%$ to photospheric/unresolved emission close to the stars, while $16 \pm 6 \%$ extended emission that is over-resolved on the shortest baselines, indicating a Gaussian half-width-athalf-maximum (HWHM) size $\gtrsim 50$ mas. We speculate that this extended $K$-band flux might trace the same physical structure that is also seen in our $L^{\prime}$-band image. The radial intensity cut through the $L^{\prime}$-band image reveals that IRAS17216-3801-A+B contribute $\lesssim 20 \%$, while the majority of the $L^{\prime}$ flux traces an extended component with a HWHM size $\sim 200$ mas (Fig. 3, bottomright). We note that this measured size is in good agreement with the expected inner truncation radius of the circumbinary disk around IRAS17216-3801-A+B. Hydrodynamic simulation suggest that binary systems with low eccentricity truncate their circumbinary disk at $\sim 2-3 \times$ the semi-major axis $a$. Using our lower limit on the semi-major axis ( $a \gtrsim 58 \mathrm{mas})$, we therefore expect the circumbinary disk to extend beyond $\gtrsim 120-180$ mas, in good agreement with the size of the $L^{\prime}$-band structure.

\subsection{Accretion-tracing and disk-tracing emission lines}

The GRAVITY wavelength-different visibilities/phases allow us to determine the distribution of the $\mathrm{Br} \gamma$ and $\mathrm{CO}$ line-emitting gas. It is interesting to note that the visibility/phase signals in $\mathrm{Br} \gamma$ and $\mathrm{CO}$ appear to be uncorrelated and are stronger in CO (despite their weaker line strength), which already indicates that these lines emerge from substantially different regions in the circumstellar environment.

The Br $\gamma$-line traces hot $\left(\gtrsim 10^{4} \mathrm{~K}\right)$ ionized gas and is a well-established mass accretion/ejection tracer (Natta et al. 2004; Kraus et al. 2012; Caratti o Garatti et al. 2016). Therefore, we model $\mathrm{Br} \gamma$ as unresolved emission at the position of the stars. Our modeling allows us to exclude scenarios that associate the $\operatorname{Br} \gamma$ only with the primary or secondary component, as this results in much stronger visibility/phase signals than measured. We achieve a satisfactory fit by attributing $40 \pm 10 \%$ of the $\operatorname{Br} \gamma$-emission to the primary and $60 \pm 10 \%$ to the secondary (Fig. 4, right).

The CRIRES spectro-astrometry provides an independent method for constraining the origin of the $\operatorname{Br} \gamma$ emission. The $\mathrm{Br} \gamma$ photocenter is displaced along $\mathrm{PA}=161 \pm$ $3^{\circ}$, which is consistent with the binary separation vector derived from the AMBER data for the same epoch $\left(159.4^{\circ}\right)$. The offset is $\sim 14 \pm 1$ mas, which places the photocenter between the two stars (Fig. 4, left), again indicating that both components contribute to the line emission. Modeling the photocenter displacement quantitatively we find that the primary (secondary) contributes $38 \%(62 \%)$ of the Br $\gamma$-flux. Therefore, the GRAVITY and CRIRES data indicate independently that the (likely lower-mass) B component accretes at a higher rate.

The CO bandhead emission is believed to trace warm $\left(\gtrsim 10^{3} \mathrm{~K}\right)$ neutral gas in the more extended disk regions (few au to a few hundred au in massive YSOs; Ilee et al. 2013). To model the GRAVITY CO line data, we approximate the CO-emitting region with a Gaussian of FWHM $\Sigma_{\mathrm{CO}}$. We vary the position of the Gaussian along the binary separation vector, where $s_{\mathrm{CO}}$ denotes the separation of the Gaussian to the primary. We find that the $\mathrm{CO}$ emission is extended $\left(\Sigma_{\mathrm{CO}}=5.8 \pm 0.8 \mathrm{mas}\right)$ and that it is centered on a position between the two stars $\left(s_{\mathrm{CO}}=31.6 \pm 3.3 \mathrm{mas}\right)$, possibly including contributions from both circumstellar disks and the gas streams between the disks (Fig. 4, right).

\section{CONCLUSIONS}



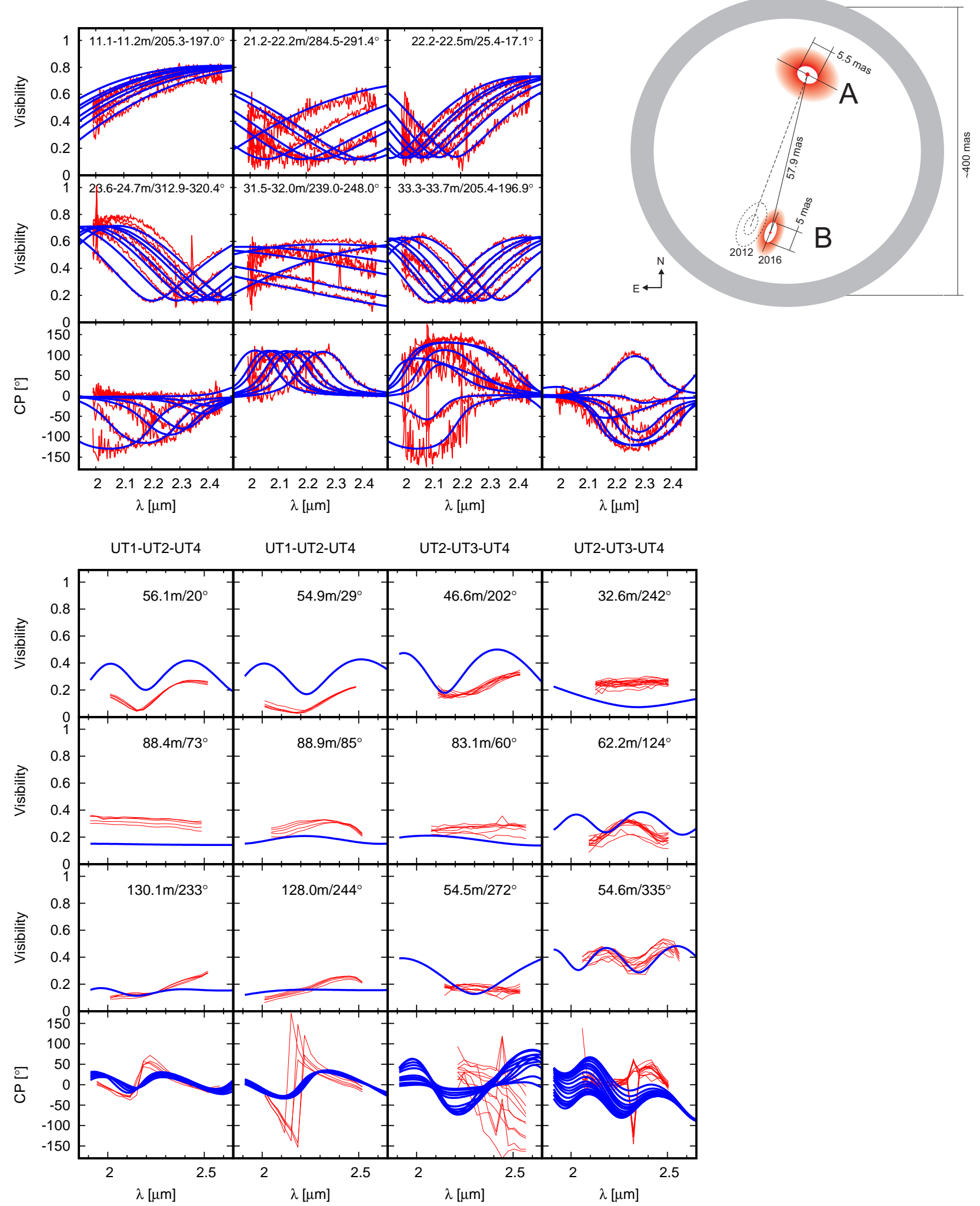

FIG. 2.- GRAVITY (top panel; red lines, 300s bins) and AMBER data (bottom panel; red lines, 50s bins), overplotted with the best-fit model (blue lines). In the top-right corner, we illustrate the model components (sketch not to scale). 
Our VLTI interferometry resolves the high-mass protostar IRAS17216-3801 into a close $(58 \mathrm{mas}=170 \mathrm{au})$ binary. The system is $\sim 3 \times$ as massive $\left(\sim 20+18 M_{\odot}\right)$ and $\sim 5 \times$ more compact than the benchmark protobinary IRAS20126+4104 (separation 850au, Sridharan et al. 2005). IRAS17216-3801 is also $\sim 3 \times$ more compact than the NGC7538 IRS1a/b system (separation 500au), where Keplerian-rotating methanol maser disks (Pestalozzi et al. 2004; Moscadelli \& Goddi 2014) and ammonia line absorption consistent with a circumbinary envelope were found (Goddi et al. 2015).

Our IRAS17216-3801 imaging traces the thermal dust emission in the circumprimary and circumsecondary disk and reveals that both disks are misaligned with respect to the binary separation vector. Various formation mechanisms have been proposed that might have resulted in the observed disk misalignments, including turbulent disk fragmentation, perturbation by a third component, star-disk capture, or infall of material whose angular momentum vector was misaligned to that of the gas that formed the binary initially (Bate et al. 2010). Once a highly misaligned system has formed, tidal interactions will work towards re-aligning the disks, which has been proposed to happen on the viscous timescale (Papaloizou \& Terquem 1995) or on the much shorter precession timescale (Bate et al. 2000). From the deduced stellar masses and outer disk radii (Tab. 1) we estimate the precession timescale of the IRAS17216-3801 circumprimary disk to $\sim 200,000$ years and $\sim 900,000$ years for the circumsecondary disk, although these values should be considered upper limits as we derive only lower limits on the disk radii from our infrared observations. Therefore, our observations indicate that the tidal realignment is still ongoing, highlighting the young dynamical age of the system.

The stronger orbital misalignment of the circumpri- mary disk might also explain its larger spatial extend, as the misalignment results in a weaker Lindblad torque that acts to truncate the circumstellar disks (Lubow et al. 2015)

The circumstellar disks are fed by a circumbinary disk/envelope that has been resolved by our VLT/NACO $L^{\prime}$-band imaging. We detect an extended structure, whose size matches roughly the expected dynamical truncation radius of the IRAS17216-3801 binary system. Both circumstellar disks show signatures of ongoing accretion, where we measure a higher $\operatorname{Br} \gamma$-line luminosity at the position of the secondary than at the primary. This suggests that the secondary disrupts the accretion stream on the primary and channels most of the infalling material onto the circumsecondary disk, confirming the prediction of hydrodynamic simulations (Bate \& Bonnell 1997). We resolved for the first time the CO line-emitting region in a massive YSO and find that it traces primarily warm neutral gas located between the two components.

With its unique properties IRAS17216-3801 provides an ideal laboratory for studying the formation of young high-mass multiple systems and to unravel how such systems accrete from their disks.

We thank the GRAVITY consortium and the Science Verification team, which is composed of ESO employees and GRAVITY consortium members (https://www.eso.org/sci/activities/vltsv/gravitysv.html). We acknowledge support from an STFC Rutherford fellowship/grant (ST/J004030/1, ST/K003445/1), Marie Sklodowska-Curie CIG grant (\#618910), Philip Leverhulme prize (PLP-2013-110), and ERC Starting grant (Grant Agreement \#639889).

Facilities: VLTI, VLT.

\section{REFERENCES}

Argon, A. L., Reid, M. J., \& Menten, K. M. 2000, ApJS, 129, 159 Bally, J., \& Zinnecker, H. 2005, AJ, 129, 2281

Bate, M.' R., \& Bonnell, I. A. 1997, MNRAS, 285, 33

Bate, M. R., Bonnell, I. A., Clarke, C. J., Lubow, S. H., Ogilvie, G. I., Pringle, J. E., \& Tout, C. A. 2000, MNRAS, 317, 773 Bate, M. R., Lodato, G., \& Pringle, J. E. 2010, MNRAS, 401, 1505

Boley, P. A., et al. 2013, A\&A, 558, A24

Bonneau, D., Delfosse, X., Mourard, D., Lafrasse, S., Mella, G.,

Cetre, S., Clausse, J.-M., \& Zins, G. 2011, A\&A, 535, A53

Caratti o Garatti, A., et al. 2016, A\&A, 589, L4

Caswell, J. L. 1998, MNRAS, 297, 215

Cesaroni, R., Neri, R., Olmi, L., Testi, L., Walmsley, C. M., \&

Hofner, P. 2005, A\&A, 434, 1039

Chelli, A., Utrera, O. H., \& Duvert, G. 2009, A\&A, 502, 705

Chiang, E. I., \& Goldreich, P. 1997, ApJ, 490, 368

Chini, R., Hoffmeister, V. H., Nasseri, A., Stahl, O., \& Zinnecker, H. 2012, MNRAS, 424, 1925

Clarke, C. J., \& Pringle, J. E. 1991, MNRAS, 249, 584

Cohen, R. J., Baart, E. E., \& Jonas, J. L. 1988, MNRAS, 231, 205

Dale, J. E., \& Davies, M. B. 2006, MNRAS, 366, 1424

Eisenhauer, F., et al. 2011, The Messenger, 143, 16

Goddi, C., Zhang, Q., \& Moscadelli, L. 2015, A\&A, 573, A108

Grellmann, R., Preibisch, T., Ratzka, T., Kraus, S., Helminiak,

K. G., \& Zinnecker, H. 2013, A\&A, 550, A82

Hofmann, K.-H., Weigelt, G., \& Schertl, D. 2014, A\&A, 565, A48

Ilee, J. D., et al. 2013, MNRAS, 429, 2960

Johnston, K. G., et al. 2015, ApJ, 813, L19

Kaeufl, H.-U., et al. 2004, in Proc. SPIE, Vol. 5492,

Ground-based Instrumentation for Astronomy, ed. A. F. M.

Moorwood \& M. Iye, 1218-1227

Kratter, K. M., \& Matzner, C. D. 2006, MNRAS, 373, 1563
Kraus, S., Calvet, N., Hartmann, L., Hofmann, K.-H., Kreplin, A., Monnier, J. D., \& Weigelt, G. 2012, ApJ, 752, 11

Kraus, S., Hofmann, K., Malbet, F., Meilland, A., Natta, A.,

Schertl, D., Stee, P., \& Weigelt, G. 2009, A\&A, 508, 787

Kraus, S., et al. 2005, AJ, 130, 246

- 2010, Nature, 466, 339

Krumholz, M. R., Klein, R. I., McKee, C. F., Offner, S. S. R., \& Cunningham, À. J. 2009, Science, 323, 754

Lapeyrere, V., et al. 2014, in Proc. SPIE, Vol. 9146, Optical and Infrared Interferometry IV, 91462D

Lenzen, R., et al. 2003, in Proc. SPIE, Vol. 4841, Instrument Design and Performance for Optical/Infrared Ground-based Telescopes, ed. M. Iye \& A. F. M. Moorwood, 944-952

Lubow, S. H., Martin, R. G., \& Nixon, C. 2015, ApJ, 800, 96

Moscadelli, L., \& Goddi, C. 2014, A\&A, 566, A150

Natta, A., Testi, L. Muzerolle, J., Randich, S., Comerón, F., \& Persi, P. 2004, A\&A, 424, 603

Papaloizou, J. C. B., \& Terquem, C. 1995, MNRAS, 274, 987

Persson, S. E., \& Campbell, B. 1987, AJ, 94, 416

Pestalozzi, M.' R., Elitzur, M., Conway, J. E., \& Booth, R. S. 2004, ApJ, 603, L113

Petrov, R. G., et al. 2007, A\&A, 464, 1

Robitaille, T. P., Whitney, B. A., Indebetouw, R., Wood, K., \& Denzmore, P. 2006, ApJS, 167, 256

Rousset, G., et al. 2003, in Proc. SPIE, Vol. 4839, Adaptive Optical System Technologies II, ed. P. L. Wizinowich \& D. Bonaccini, 140-149

Sridharan, T. K., Williams, S. J., \& Fuller, G. A. 2005, ApJ, 631, L73

Tatulli, E., et al. 2007, A\&A, 464, 29

Yorke, H. W., \& Sonnhalter, C. 2002, ApJ, 569, 846 

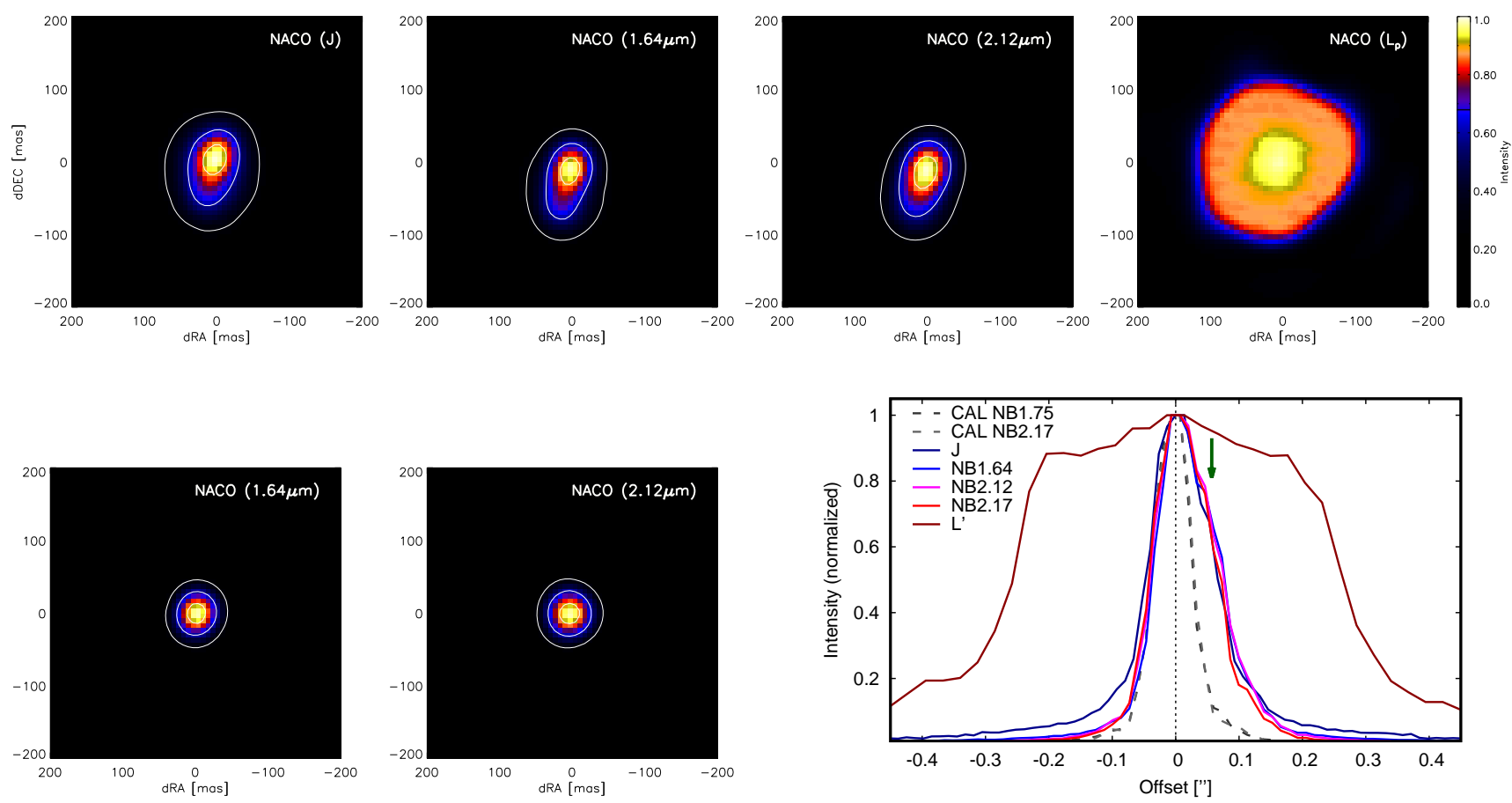

FIG. 3.- NACO imaging of IRAS17216-3801 (top) and standard star HD155974 (bottom-left). For the J-band $/ 1.64 \mu \mathrm{m} / 2.12 \mu \mathrm{m}$ image, we plot intensity contours at $30 \%, 60 \%$, and $90 \%$ of peak intensity (white contours). The bottom right panel shows radial intensity cuts along the binary separation vector from IRAS17216-3801 (solid lines) and the standard star images (dashed lines). The arrow indicates the position of IRAS17216-3801-B, as derived from interferometry. 

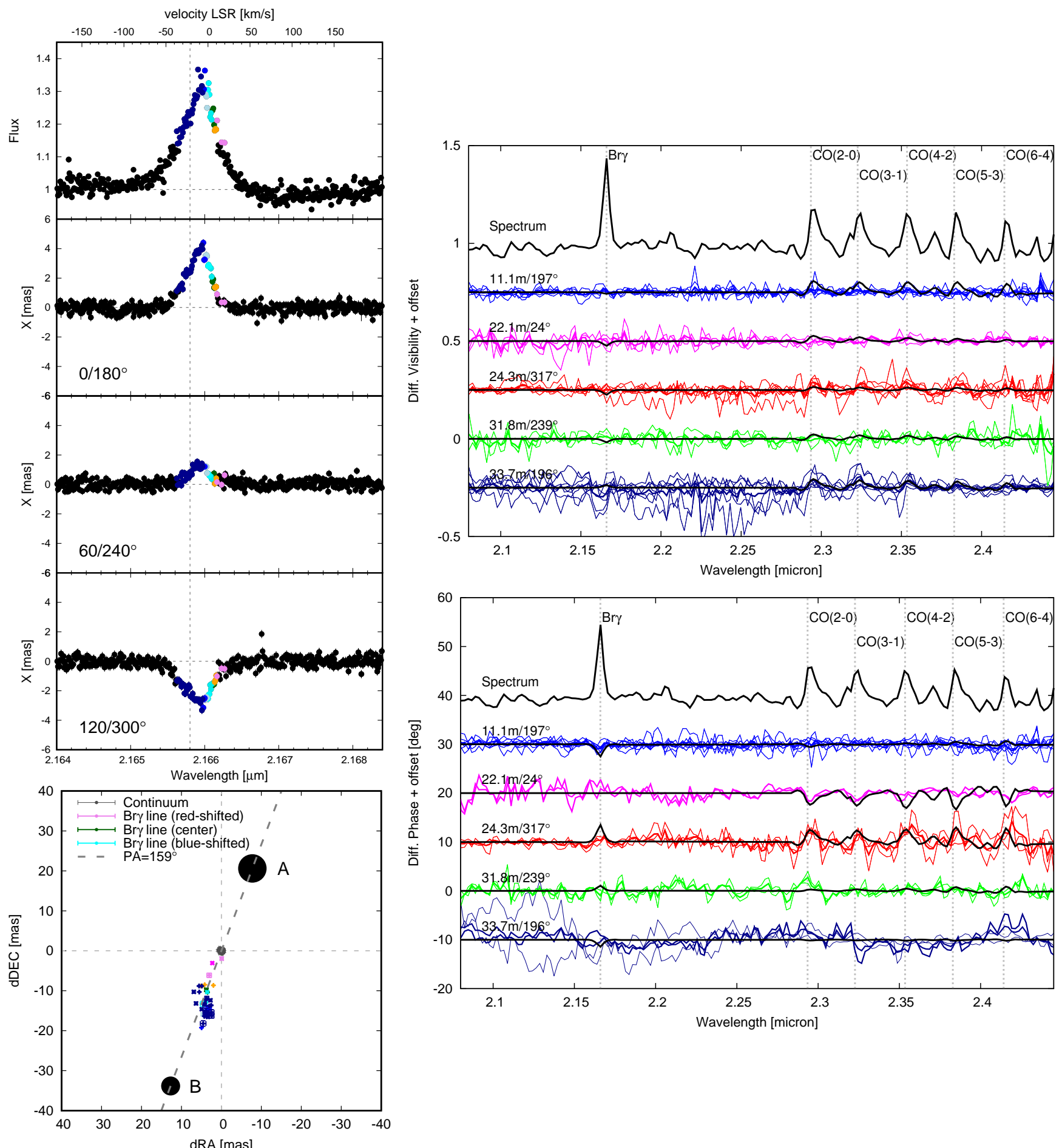

FIG. 4.- CRIRES spectro-astrometric (left) and GRAVITY spectro-interferometric data (right) on IRAS17216-3801. Top-left: The $\mathrm{Br} \gamma$ line is single-peaked and shows strong astrometric signals (X) toward different PAs. Bottom-left: Continuum-corrected 2-dimensional photocenter offsets derived from the astrometric signals, with the color indicating the wavelength of the corresponding channel. For clarity, we also indicated the position of IRAS17216-3801-A+B, as derived with GRAVITY+AMBER (Sect. 4.2). Right: Spectra, differential phases, and differential visibilities derived from our GRAVITY data. The black lines show the visibilities/phases that correspond to the $\mathrm{Br} \gamma / \mathrm{CO}$ model outlined in Sect. 4.3 